\begin{tabular}{|c|c|c|}
\hline$T$ & $\begin{array}{l}\text { European Association for the } \\
\text { Development of Renewable Energies, Environment } \\
\text { and Power Quality (EA4EPQ) }\end{array}$ & $\begin{array}{l}\text { International Conference on Renewable Energies and Power Quality } \\
\text { (ICREPQ'12) } \\
\text { Santiago de Compostela (Spain), 28th to 30th March, } 2012\end{array}$ \\
\hline
\end{tabular}

\title{
Voltage Regulation in Low Voltage Distribution Networks with Embedded Photovoltaic Microgeneration
}

\author{
Marcelo Pais, M.E. Almeida and Rui Castro \\ Centre for Innovation in Electrical and Energy Engineering (Cie3) \\ IST - Technical University of Lisbon, Portugal \\ Av. Rovisco Pais, 1049-011 Lisboa (Portugal)

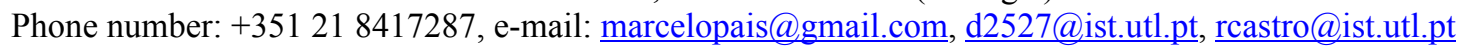

\begin{abstract}
Low voltage distribution networks have been originally designed to feed loads. However, recently, small dispersed renewable generators, mainly photovoltaics, are being connected to the low voltage grids. This new operational framework represents a challenge to the planning and operation of distribution networks, since the multidimensional stochastic dependence structure of the joint behaviour of the generation and load can be very complex to model. This paper intends to give further insight to the subject by presenting a three-phase unbalanced load flow method suitable to deal with radial networks with embedded microgeneration. Some results concerning the voltage profile as obtained after Monte-Carlo simulations for different load scenarios are presented in the paper, as well as an assessment of the generation shedding procedures undertaken to control the voltage.
\end{abstract}

Keywords: Voltage regulation, Low voltage networks, Photovoltaic microgeneration, Three-phase load flow, Monte-Carlo simulation.

\section{Framework}

It is a fact that little efforts have been made so far to reduce the net load as seen from the electrical system. Assuming that the straightforward reduction of the rate of electricity consumption is not easily compatible with the development and well-fare of the populations, the same target can be accomplished in an indirect way, by producing locally part of the electrical energy consumed and thus providing some local compensation. The use of renewable energy sources to produce that electrical energy would turn the idea in an even better one. That is why, recently, governments are encouraging the installation of small urban PhotoVoltaic (PV) units (usually in the roofs of the buildings), typically sized in the range of some $\mathrm{kW}$, in the framework of the so called microgeneration.

The microgeneration consists of a combination of generation sources, usually renewable, that are interfaced with the low voltage distribution network through fast acting power electronics. From the point of view of the low voltage distribution networks, which have been designed to feed loads, this is a quite innovative aspect. As a consequence, the power flow between the transmission network and the now active distribution networks is no longer unidirectional but, actually, it can be bidirectional. The power can flow "vertically", from higher levels to the lower voltage levels, or vice-versa, and also "horizontally", from one distribution network to another or from a generator to a load within the same distribution network.

As far as the conventional load flow problem in the high voltage transmission network, or even in the medium voltage distribution network, is concerned, the power system is assumed to be balanced. Therefore, a singlephase positive sequence analysis can be performed and holds true. This is not, however, the case of the low voltage distribution network, which is inherently unbalanced due to the unbalance of the domestic loads. The technical solution to this case is the so called threephase load flow, in which the single phase equivalent circuit does not apply and the behaviour of each phase must be individually considered [1-5].

The three-phase load flow in a context of unbalanced loads is nowadays currently used, and the algorithms to solve it are well known and fully documented. That is not the case, however, if single-phase local generation is to be considered. There is a scarce knowledge of the behaviour of the low voltage distribution network in presence of concurrent unbalanced generation and loads [6-8]. The multidimensional stochastic dependence structure of the joint behaviour of the generation and load can be very complex to model. Furthermore, in low voltage distribution networks both three-phase and single-phase equipments can be found, which turns the problem into an even more complex one.

The objective of this paper is twofold: on one hand, to assess the performance of a low voltage distribution network with embedded microgeneration, namely in what concerns the voltage profile; on the other hand, to analyse the impact of load and generation shedding as a solution to improve the voltage profile whenever it is required. 
In order to reach these objectives, a three-phase load flow algorithm, appropriate for use in radial networks, has been developed. In this algorithm, which is based on the superposition method, the branch currents are evaluated taking into account the contribution of all loads/microgenerators and considering pre-defined bus voltages. Imposing the voltage specified at the feeder bus and using the calculated branch currents one can determine the bus voltages of the network. An iterative process is used to obtain convergence and evaluate the voltages at each and every bus.

The developed algorithm is applied to a test network, so that some results concerning the voltage profile as obtained with unbalanced generation and load can be found. To take account for the random nature of the involved variables, a Monte-Carlo simulation has been carried out. Whenever the voltage rise/drop is outside the acceptable voltage limits, generation/load shedding has to be undertaken. The results of the application of these procedures are also presented and commented in the paper.

\section{Methodology}

The methodology developed is suitable for unbalanced three-phase radial distribution systems and is based on the superposition method to determine the network branch currents. As the branch currents are evaluated from the computed bus voltages, an iterative process is needed to obtain the network voltages final solution.

Three main steps can be defined to describe the algorithm developed.

The phase voltages are predefined at some initial values on the buses with load or with microgeneration and the first step of the algorithm is to determine the branch currents, starting from the end buses to the source (feeder bus, where is located the service transformer). The current in each branch is obtained adding the contribution of each load/generator at a time. The return conductor current is also obtained in order to determine the neutral voltage.

As the voltage is specified at the feeder bus, the second step consists of determining the new bus voltages using the branch currents previously calculated, as well as the neutral voltage. The calculations in this step start from the source to the end buses. In this step, the computed bus voltage corresponds to the phase-neutral voltage.

The third step is a check for convergence, where the new voltages are compared to the previous ones. If the differences are greater than a specified value, all process must be repeated from step one to step three until convergence is reached.

This methodology can be better understandable with a simple example, using a balanced network with 4-bus and 2-load, as represented in Fig.1. The service transformer, which converts medium to low voltage (MV/LV), is connected to bus 1 .

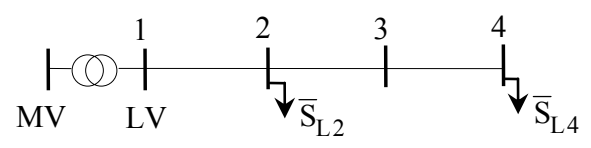

Fig. 1. Balanced network diagram used on the illustration example.
We assume that the branches are represented by its longitudinal impedance and, at start, the bus voltages are equal to the specified voltage at the feeder bus $\left(\overline{\mathrm{V}}_{\mathrm{i}}^{0}=\overline{\mathrm{V}}_{1}\right)$.

The first step is to determine the branch currents, starting from bus 4 to bus 1 , considering the contribution of each load at a time, as shown in Fig.2.

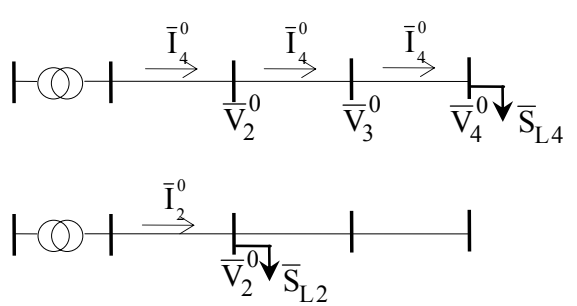

Fig. 2. Contribution of each load to obtain the branch currents: first step methodology application; balanced network.

Firstly, we compute the currents related to each load at a time as:

$$
\begin{aligned}
& \overline{\mathrm{I}}_{4}^{0}=\left(\overline{\mathrm{S}}_{\mathrm{L} 4} / \overline{\mathrm{V}}_{4}^{0}\right)^{*} \\
& \overline{\mathrm{I}}_{2}^{0}=\left(\overline{\mathrm{S}}_{\mathrm{L} 2} / \overline{\mathrm{V}}_{2}^{0}\right)^{*}
\end{aligned}
$$

The branch currents due to the contribution of each load are:

$$
\begin{aligned}
& \overline{\mathrm{I}}_{12}^{0}=\overline{\mathrm{I}}_{2}^{0}+\overline{\mathrm{I}}_{4}^{0} \\
& \overline{\mathrm{I}}_{23}^{0}=\overline{\mathrm{I}}_{34}^{0}=\overline{\mathrm{I}}_{4}^{0}
\end{aligned}
$$

New bus voltages are evaluated in a second step using the specified voltage at feeder bus and the previously calculated branch currents as:

$$
\begin{aligned}
& \overline{\mathrm{V}}_{2}^{1}=\overline{\mathrm{V}}_{1}-\overline{\mathrm{Z}}_{12} \overline{\mathrm{I}}_{12}^{0} \\
& \overline{\mathrm{V}}_{3}^{1}=\overline{\mathrm{V}}_{2}^{1}-\overline{\mathrm{Z}}_{23} \overline{\mathrm{I}}_{23}^{0} \\
& \overline{\mathrm{V}}_{4}^{1}=\overline{\mathrm{V}}_{3}^{1}-\overline{\mathrm{Z}}_{34} \overline{\mathrm{I}}_{34}^{0}
\end{aligned}
$$

On a third step, this new voltages (with superscript 1) are compared to the previous predefined values (with superscript 0 ). If the differences are greater than a specified value, all process must be repeated from step one (using now the new voltages) to step three until convergence is reached.

For an unbalanced network, let us consider the network represented in Fig.3, where $\overline{\mathrm{S}}_{\mathrm{L} 2}$ is a load connected in bus 2, between phase $\mathrm{C}$ and the neutral (wire not represented in this figure), and $\overline{\mathrm{S}}_{\mathrm{L} 4}$ is a load connected in bus 4 , between phase A and the neutral.

At start, we consider that bus phase-neutral voltages are balanced and equal to the specified voltage at the feeder bus $\left(\overline{\mathrm{V}}_{\mathrm{A}_{\mathrm{i}}}^{0}=\overline{\mathrm{V}}_{\mathrm{A}_{1}}, \overline{\mathrm{V}}_{\mathrm{B}_{\mathrm{i}}}^{0}=\overline{\mathrm{V}}_{\mathrm{B}_{1}}\right.$, and $\left.\overline{\mathrm{V}}_{\mathrm{C}_{\mathrm{i}}}^{0}=\overline{\mathrm{V}}_{\mathrm{C}_{1}}\right)$. 


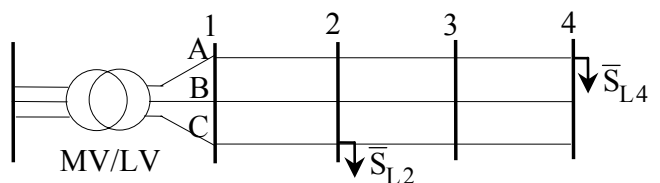

Fig. 3. Unbalanced network used on the illustration example.

On a first step, the phase currents are evaluated, starting from bus 4 to bus 1 , considering the contribution of each load at a time, as shown in Fig.4. Currents $\overline{\mathrm{I}}_{\mathrm{A}}^{0}$ and $\overline{\mathrm{I}}_{\mathrm{C}}^{0}$ are computed using an equation similar to (1), being the phase and neutral branch currents given by:

$$
\begin{array}{ll}
\overline{\mathrm{I}}_{\mathrm{A}_{12}}^{0}=\overline{\mathrm{I}}_{\mathrm{A}}^{0} & \overline{\mathrm{I}}_{\mathrm{A}_{23}}^{0}=\overline{\mathrm{I}}_{\mathrm{A}_{34}}^{0}=\overline{\mathrm{I}}_{\mathrm{A}}^{0} \\
\overline{\mathrm{I}}_{\mathrm{B}_{12}}^{0}=0 & \overline{\mathrm{I}}_{\mathrm{B}_{23}}^{0}=\overline{\mathrm{I}}_{\mathrm{B}_{34}}^{0}=0 \\
\overline{\mathrm{I}}_{\mathrm{C}_{12}}^{0}=\overline{\mathrm{I}}_{\mathrm{C}}^{0} & \overline{\mathrm{I}}_{\mathrm{C}_{23}}^{0}=\overline{\mathrm{I}}_{\mathrm{C}_{34}}^{0}=0 \\
\overline{\mathrm{I}}_{\mathrm{N}_{12}}^{0}=-\overline{\mathrm{I}}_{\mathrm{A}}^{0}-\overline{\mathrm{I}}_{\mathrm{C}}^{0} & \overline{\mathrm{I}}_{\mathrm{N}_{23}}^{0}=\overline{\mathrm{I}}_{\mathrm{N}_{34}}^{0}=-\overline{\mathrm{I}}_{\mathrm{A}}^{0}
\end{array}
$$

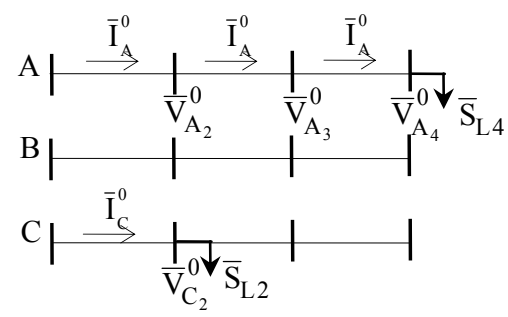

Fig. 4. Contribution of each load to obtain the branch currents: first step methodology application; unbalanced network.

On a second step, new phase to neutral voltages are evaluated using the specified voltage at feeder bus and the previously computed branch currents as:

$\overline{\mathrm{V}}_{\mathrm{k}_{2}}^{1}=\overline{\mathrm{V}}_{\mathrm{k}_{1}}-\overline{\mathrm{Z}}_{\mathrm{k}_{12}} \overline{\mathrm{I}}_{\mathrm{k}_{12}}^{0}+\overline{\mathrm{Z}}_{\mathrm{N}_{12}} \overline{\mathrm{I}}_{\mathrm{N}_{12}}^{0}$

$\overline{\mathrm{V}}_{\mathrm{k}_{3}}^{1}=\overline{\mathrm{V}}_{\mathrm{k}_{2}}^{1}-\overline{\mathrm{Z}}_{\mathrm{k}_{23}} \overline{\mathrm{I}}_{\mathrm{k}_{23}}^{0}+\overline{\mathrm{Z}}_{\mathrm{N}_{23}} \overline{\mathrm{I}}_{\mathrm{N}_{23}}^{0}$

$\overline{\mathrm{V}}_{\mathrm{k}_{4}}^{1}=\overline{\mathrm{V}}_{\mathrm{k}_{3}}^{1}-\overline{\mathrm{Z}}_{\mathrm{k}_{34}} \overline{\mathrm{I}}_{\mathrm{k}_{34}}^{0}+\overline{\mathrm{Z}}_{\mathrm{N}_{34}} \overline{\mathrm{I}}_{\mathrm{N}_{34}}^{0}$

The subscript $\mathrm{k}$ is related to the phase under analysis (A, B or $\mathrm{C}$ ). This simplified analysis neglects mutual impedances between different conductors and assumes the neutral voltage at service transformer equal to zero.

The third step is similar to the one described for the balanced network. The new voltages (with superscript 1) are compared to the previous predefined values (with superscript 0). If the differences are greater than a specified value, all process must be repeated from step one (using now the new voltages) to step three until convergence is reached.

\section{Case Study Definition}

In order to apply the developed methodology, the following 12-bus test network has been considered.

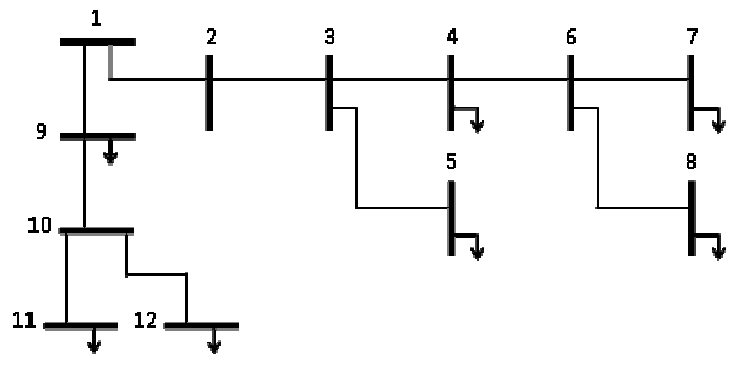

Fig. 5. Test network diagram.

A Medium Voltage / Low Voltage (MV/LV) $250 \mathrm{kVA}$ transformer is connected to busbar 1 , where the voltage is considered to be constant and equal to $1.05 \mathrm{pu}$. All the cables are equal with an $\mathrm{R} / \mathrm{X}$ ratio equal to 20 .

In what concerns the load, two scenarios have been considered: (i) a load peak scenario, in which the unbalanced loads represent a night maximum load; for this case, the base load at each bus was set to $10+\mathrm{j} 5$ [kW/kvar]; (ii) a load valley scenario, in which the unbalanced loads represent a minimum daylight load; this valley load was set equal to one third of the base peak load.

In the load valley scenario, microgeneration has been included to represent PV embedded generation. This PV generation is located in the load buses, is single-phased and its value is equal to half the base maximum load in that particular bus.

In order to account for the random nature of both the generation and the load, a Monte-Carlo simulation, with 10000 runs, has been carried out. This objective was accomplished by assigning uniform random variables to:

1) Load

(i) The distribution percentage of the load between the three phases (total of $100 \%$ in the three phases).

(ii) The total load percentage (between $80 \%$ and $100 \%$ of the maximum) in each bus.

2) PV Generation

(i) The connection phase of the single phased generation (phase A, B or C).

(ii) The generation percentage (between $80 \%$ and $100 \%$ of the maximum) in each bus.

\section{Results and Discussion}

Two separated Monte-Carlo simulations have been carried out for the load peak night scenario (without microgeneration) and for the load valley daylight scenario (with microgeneration).

\section{A. Load peak scenario}

This scenario aims at simulating a night situation, in which the load is at its maximum value and no PV microgeneration is found.

It has been considered that the load at each bus and at each run of the Monte-Carlo simulation may fluctuate between $80 \%$ and $100 \%$ of the base value. This was achieved by means of a uniformly distributed random 
variable. Also, uniform random variables were used to distribute the load among the three phases, for each bus and for each run of the Monte-Carlo simulation.

After the 10000 runs of the Monte-Carlo simulation, very rare situations in which the voltage is above $1.1 \mathrm{pu}$ occurred. On the other hand, several situations in which the voltage is under $0.9 \mathrm{pu}$ were found. The probability of the voltage being less than $0.9 \mathrm{pu}$ is depicted in Fig.6.

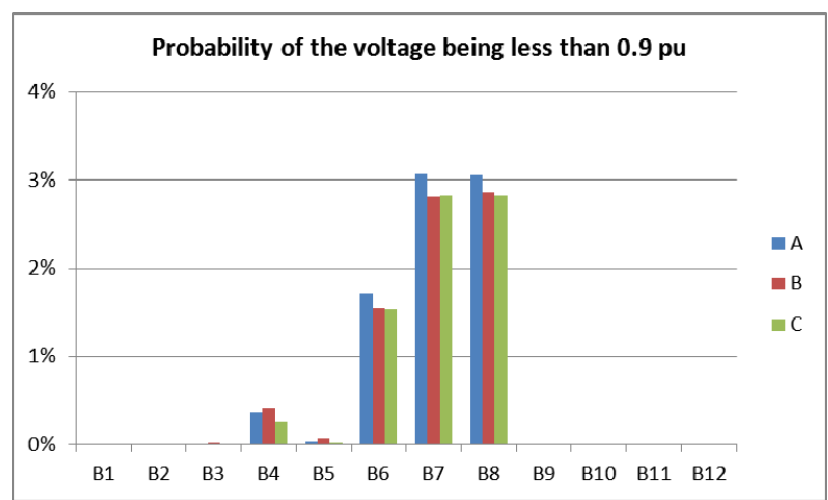

Fig. 6. Probability of the voltage being less than $0.9 \mathrm{pu}$, load peak scenario

From Fig.6 one can observe that the probability of the voltage being less than $0.9 \mathrm{pu}$ is relatively small. The greater values (around 3\%) are obtained in busbars 7 and 8 , which are the ones that are far away from the transformer. This result is due to the fact that the voltage at busbar 1 is set equal to $1.05 \mathrm{pu}$, so in most situations the voltage is kept under acceptable limits along all the network.

The worst case, in which the voltage in busbars 7 and 8 dropped under $0.75 \mathrm{pu}$, was obtained with a rather unbalanced load as can be seen in Fig.7.

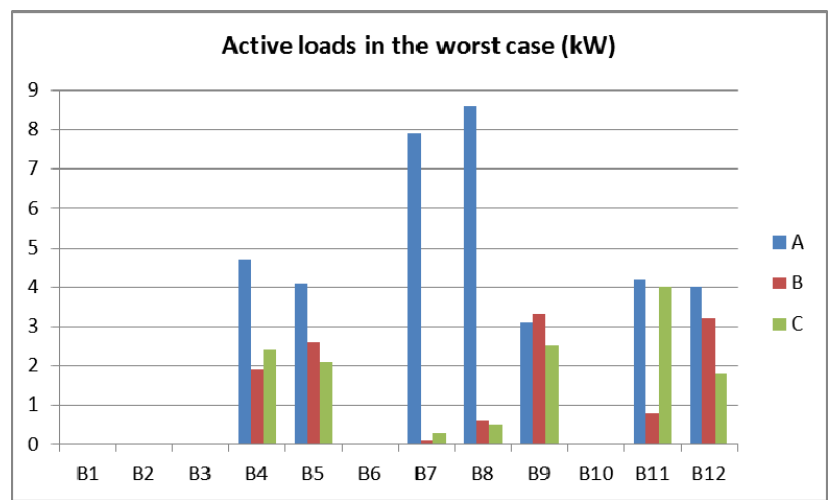

Fig. 7. Phase active loads in the worst case, load peak scenario

Another network unbalance indicator is the neutral voltage, which can be seen in Fig. 8 for the worst case.

The probability of having at least one phase bus voltage under $0.9 \mathrm{pu}$ is around $10 \%$. If load shedding is chosen as a voltage control strategy, a dedicated study has shown that removing the load located in the bus with the lowest voltage would solve the problem, in most situations.

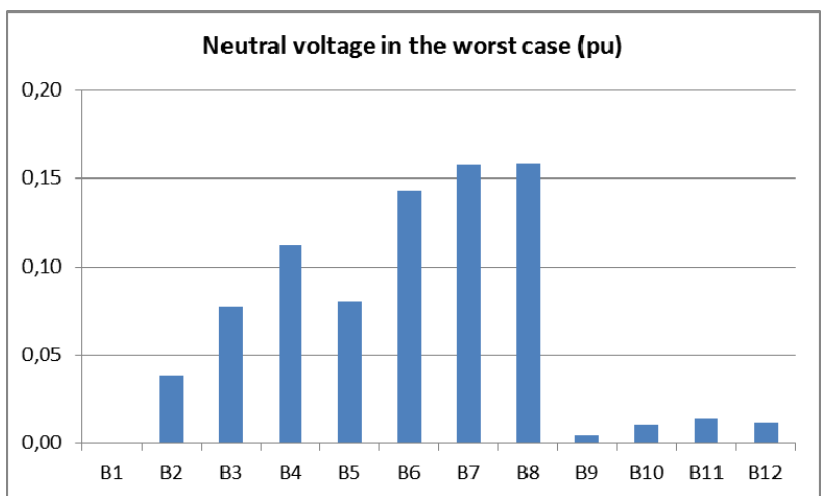

Fig. 8. Neutral voltage in the worst case, load peak scenario

\section{B. Load valley scenario}

This scenario aims at simulating a daylight situation, in which the load is at its minimum value and PV microgeneration is injecting active power in the network. As it was done in the load peak scenario, the load at each bus and at each run of the Monte-Carlo simulation may fluctuate between $80 \%$ and $100 \%$ of the valley value, which we recall is set equal to one third of the base value. Also, this load was distributed among the three phases using uniform random variables.

In what concerns the PV generators, they are located in each load bus and are sized half of the base maximum load. A uniform random variable is used to select the injected power between $80 \%$ and $100 \%$. This procedure aims at accounting for changing sun irradiance conditions. As PV microgeneration is single-phased, a uniform random variable was used to select the particular connection phase, for each bus and for each Monte-Carlo run.

The results obtained after the 10000 simulations for the load valley scenario are presented in Fig.9, which concerns the probability of the voltage being greater than $1.1 \mathrm{pu}$, for each phase and for each bus.

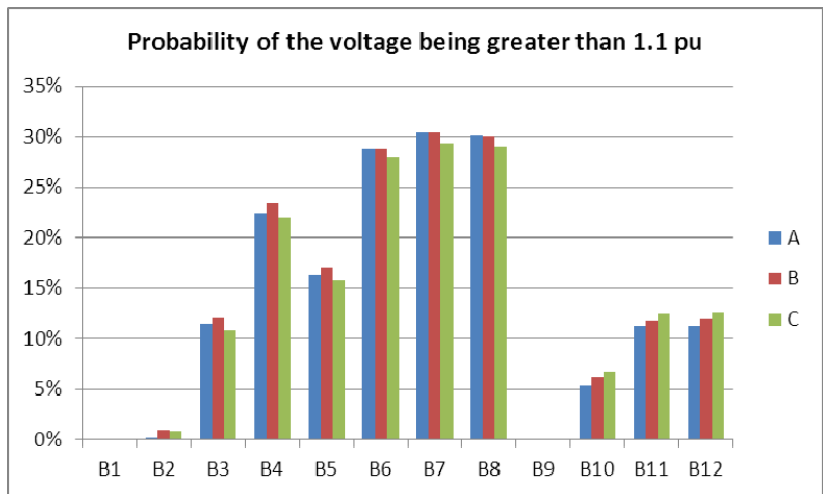

Fig. 9. Probability of the voltage being greater than $1.1 \mathrm{pu}$, load valley scenario

No situations in which the voltage is less than $0.9 \mathrm{pu}$ were expected to occur and in fact they did not occur. However, as reported in Fig.9, the probability that the voltage is greater than $1.1 \mathrm{pu}$ can reach around $30 \%$ in buses 7 and 8, which are the most sensible ones.

It should be recalled that the voltage at bus 1 has been set to 1.05 , which is a standard practise to face the voltage drop in the load peak scenario. This procedure is largely responsible for the detected overvoltages. 
In order to solve this overvoltage problem, three possible solutions have been assessed and are discussed hereafter.

\section{Solution 1 - MV/LV transformer tap changing}

Usually, the $\mathrm{MV} / \mathrm{LV}$ transformer does not possess automatic load tap changing capabilities. Instead, an appropriate tap should be selected with the transformer off service. Till now, this fixed tap was chosen so that the voltage along the entire feeder is above the lower limits in peak load hours (off-peak generation hours). However, with embedded microgeneration, the fixed tap should also guarantee that the voltage along the feeder is under the upper limits in off-peak load hours (peak generation hours). This twofold objective can be hard to achieve.

In order to assess this issue, a Monte-Carlo simulation was carried out with the voltage at the MV/LV transformer (bus 1) equal to $1.0 \mathrm{pu}$. The results are shown in Fig.10, for the load valley scenario.

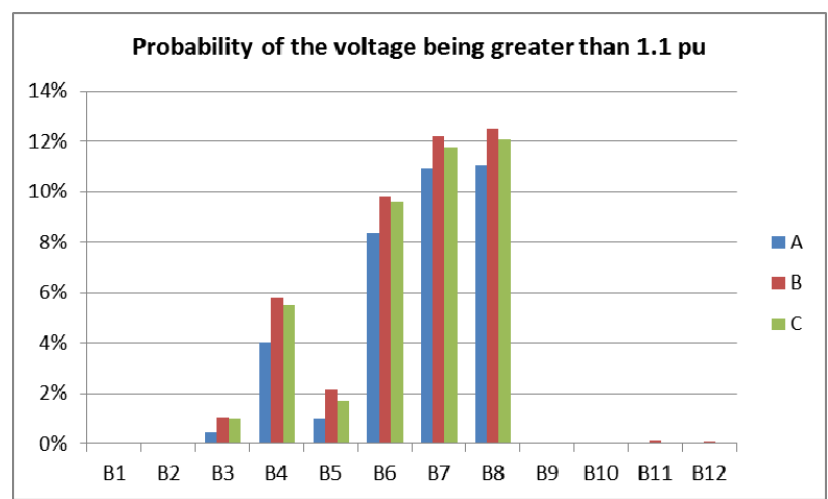

Fig. 10. Probability of the voltage being greater than $1.1 \mathrm{pu}$, load valley scenario, $\mathrm{V}_{1}=1.0 \mathrm{pu}$

From Fig.10, it is noticeable that a significant reduction in the probability of the voltage being above $1.1 \mathrm{pu}$ was achieved. However, one should bear in mind that this tap changing procedure has a non-negligible impact in the voltage profile in the peak load scenario, which should be assessed. An increase in the probability of undervoltages is expected to occur in this situation.

\section{Solution 2 - PV generators power factor control}

Traditionally, the voltage control issue is dealt with by an appropriate reactive power flow control. This can be locally performed by the inverters of the PV microgenerators, which hold power factor regulation capabilities. However, it is expected that this measure is ineffective, due to the low inductive component of the LV cables.

In order to evaluate this solution, a Monte-Carlo simulation was conducted, for the load valley scenario, considering that the PV generators inverters are regulated so that there is reactive power consumption equal to $20 \%$ of the injected active power. This can be easily accommodated by the inverters control system. There is no need to show the obtained results concerning the probability of the voltage being greater than $1.1 \mathrm{pu}$, because the overall improvement is marginal: around $1.1 \%$. Furthermore, if the reactive power consumption is equal to $40 \%$ of the injected active power, the improvement would be $1.7 \%$.

\section{Solution 3 - PV generation shedding}

Generation shedding seems to be the only viable solution to control the voltage under acceptable limits.

To attain this objective, the PV generator located at the bus with the larger overvoltage is firstly removed and this procedure is repeated till all bus voltages are under $1.1 \mathrm{pu}$.

The results obtained are shown in Fig.11.

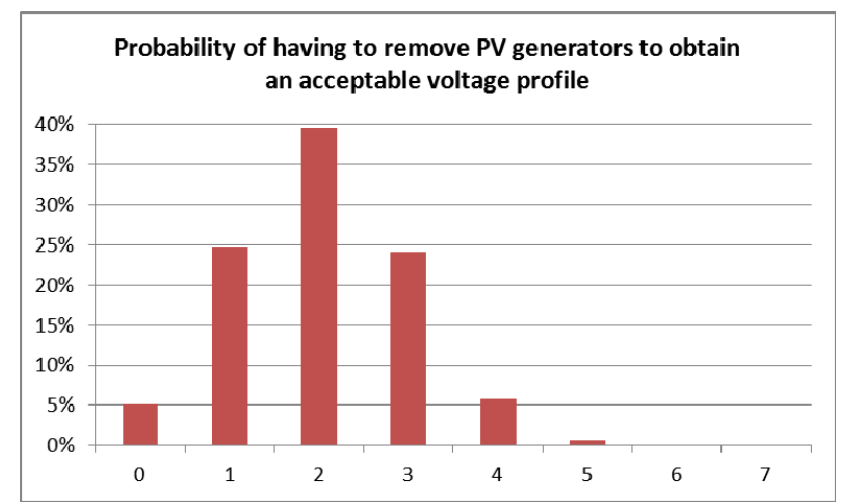

Fig. 11. Probability of having to remove PV generators to obtain an acceptable voltage profile, load valley scenario

Examining Fig. 11 one can conclude that only in 5\% of the times, the voltage profile of the network is acceptable and no PV generation shedding is required as all the voltages are under $1.1 \mathrm{pu}$. Usually, removing two PV generators is enough to keep the voltage under control along the entire network. In some extreme unbalanced cases, removing five generators is required to have an acceptable voltage profile.

\section{Conclusions}

In this paper, an unbalanced three-phase load flow method was presented. This methodology is fitted for unbalanced three-phase radial distribution systems. The basic principle behind the developed method is the superposition method, which is used to compute the network branch currents considering the contribution of each load at a time. This is initially accomplished stating that the bus voltages are equal to the swing bus voltage. Then, new phase to neutral voltages are calculated using both the phase and neutral impedances and the phase and neutral currents just computed. As the branch currents are evaluated from the computed bus voltages, an iterative process is needed to obtain the network voltages final solution.

This method was tested in a 12-buses radial distribution network with unbalanced load and microgeneration through a Monte-Carlo simulation. It was found that in a load peak night scenario, with no PV generation, the voltage profile can be very low, requiring some load shedding to rise up the voltage. On the other hand, in a load valley daylight scenario, with PV generation, the voltage profile is generally high, which requires some generation shedding to re-establish the voltage values within acceptable limits. Methodologies to determine the proper load/generation shedding order were presented in the paper. 


\section{Acknowledgement}

Prof. Pedro Carvalho from IST/TUL is deeply acknowledged for his valuable remarks and profitable discussions concerning the subject of this paper.

\section{References}

[1] M. Monfared, A.M. Daryani, M. Abedi, (2006), "Three Phase Asymmetrical Load Flow for Four-Wire Distribution Networks", 2006 IEEE PES Power Systems Conference and Exposition, (PSCE'06), Atlanta.

[2] M. Ciric, A. Feltrin, L. Ochoa, (2003), "Power Flow in Four Wire Distribution Networks - General Approach", IEEE Transactions on Power Systems, Vol.16, No.4.

[3] J.H. Teng, C.Y. Chang, (2002), "A Novel and Fast ThreePhase Load Flow for Unbalanced Radial Distribution Systems", IEEE Transactions on Power Systems, Vol.17, No.4.
[4] M. Chindriş, A.S. Anderu, C. Bud, B. Tomoiaga, (2007), "The Load Flow Calculation in Unbalanced Radial Electric Networks with Distributed Generation”, 9th International Conference on Electrical Power Quality and Utilization, (EPQU'2007), Barcelona.

[5] M. A. Golkar, (2007), "A Novel Method for Load Flow Analysis of Unbalanced Three-Phase Radial Distribution Networks", Turkish Journal of Electrical Engineering \& Computer Sciences, Vol.15, No.3.

[6] P.M.S. Carvalho, P.F. Correia, L.A.M. Ferreira, (2008), "Distributed Reactive Power Generation Control for Voltage Rise Mitigation in Distribution Networks", IEEE Transactions on Power Systems, Vol.28, No.2.

[7] M. Thomson, D.G. Infield, (2007), "Impact of Widespread Photovoltaics Generation on Distribution Systems", IET Renewables Power Generation, Vol.1, Iss.1.

[8] M. Thomson, D.G. Infield, (2007), "Network Power-Flow Analysis for a High Penetration of Distributed Generation", IEEE Transactions on Power Systems, Vol.22, No.3. 